\title{
Initial absence of N20 waveforms from median nerve somatosensory evoked potentials in a patient with cardiac arrest and good outcomes
}

\author{
Miguel E. Habeych ${ }^{1,2}$, Pouria Moshayedi ${ }^{3}$, Jon C. Rittenberger ${ }^{4}$, \\ Scott R. Gunn ${ }^{4,5}$ \\ 'Department of Anesthesiology, University of Cincinnati, Cincinnati, $\mathrm{OH}$, USA \\ ${ }^{2}$ Intraoperative Neurophysiological Monitoring Group, Cincinnati Children's Hospital Medical Center, \\ Cincinnati, $\mathrm{OH}, \mathrm{USA}$ \\ ${ }^{3}$ Department of Neurology, University of Pittsburgh, Pittsburgh, PA, USA \\ ${ }^{4}$ Department of Emergency Medicine, University of Pittsburgh, Pittsburgh, PA, USA \\ ${ }^{5}$ Department of Critical Care, University of Pittsburgh, Pittsburgh, PA, USA
}

A 34-year-old male was brought to the hospital with a chest gunshot wound. Pulseless upon arrival, blood pressure was absent for 10 minutes. A thoracotomy resulted in return of spontaneous circulation. On hospital day 5, with brainstem reflexes present, he was unresponsive to call or pain, exhibited generalized hyperreflexia and bilateral Babinskys. Median nerve somatosensory evoked potentials (mSSEPs) and brainstem auditory evoked potentials were obtained. International Federation of Clinical Neurophysiology recommendations for mSSEPs and brainstem auditory evoked potentials were followed. Despite absence of the N20 responses from cortical mSSEPs no withdrawal from care was agreed upon. After awaking on day 7, mSSEPs were repeated and present. The patient survived and was discharged with minor deficits. Bilateral absence of N2O responses from mSSEPs performed beyond 48 hours after resuscitation from cardiac arrest is highly associated with bad neurological outcomes. However, variation due to hypothermia, noisy signals, medications, and brain hypo-perfusion must be taken into account.

Keywords Evoked potentials, somatosensory; Critical care outcomes; Nervous system diseases; Prognosis

Capsule
Wummat is already known
Bilateral absence of N2O responses from cortical median nerve somatosensory
evoked potentials (mSSEPS) has been reported to be associated with death or
bad neurological outcomes after cardiac arrest. Bilateral absence of N20 re-
sponses from cortical mSSEPs can occur with survival and/or good neurological
outcome in reversible hypoglycemic coma, hypothermia, viral encephalitis, car-
bamazepine intoxication, head trauma and hemispheric infarctions.
What is new in the current study
$\begin{aligned} & \text { Bilateral absence of N20 responses from cortical mSSEPs can also be produced } \\ & \text { by brain hypo-perfusion and be associated with survival or good neurological } \\ & \text { outcome. Bilateral absence of N20 responses from cortical mSSEPs probably } \\ & \text { should be used in conjunction with other data for outcome prognostication in } \\ & \text { individual subjects after cardiac arrest. }\end{aligned}$

eISSN: 2383-4625

Received: 9 March 2018

Revised: 12 May 2018

Accepted: 24 May 2018

Correspondence to: Miguel E. Habeych Department of Anesthesia, University of Cincinnati, 3333 Burnett Avenue MLC-2001, Cincinnati, OH 452293026, USA

E-mail: miguelhabeych@gmail.com ORCID

http://orcid.org/0000-0002-4598-9540

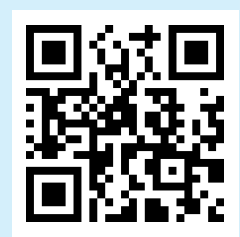

How to cite this article:

Habeych ME, Moshayedi P, Rittenberger JC, Gunn SR. Initial absence of N20 waveforms from median nerve somatosensory evoked potentials in a patient with cardiac arrest and good outcomes. Clin Exp Emerg Med 2019;6(2):177-182.

This is an Open Access article distributed under the terms of the Creative Commons Attribution Non-Commercial License (http:// creativecommons.org/licenses/by-nc/4.0/). 


\section{INTRODUCTION}

Clinicians are often faced with the scenario of predicting outcome in patients resuscitated from cardiac arrest. A recent metaanalyses demonstrated cortical, short latency somatosensory evoked potentials from median nerves (mSSEPs) are an independent factor associated with outcomes. ${ }^{1}$ Also, the bilateral absence of the N20 response from cortical mSSEPs performed within the first week of resuscitation following cardiac arrest is an accurate predictor of death or poor neurologic outcome with close to 100\% specificity. ${ }^{2}$ However, in the hypothermia era, their false positives may be as high as 3\% questioning their validity. Previous case reports have challenged the prognostic value of mSSEPs in isolation. ${ }^{3}$

Short latency auditory evoked potentials have been used to evaluate brainstem function. The neural generators of their components are dispersed along this structure, and are sequentially activated when the cochlear cranial nerve is stimulated with sound at the ears. These brainstem auditory evoked potentials (BAEPs) are more resistant than mSSEPs to sedatives and anesthetics. ${ }^{4}$ However, BAEPs have not demonstrated the same prognostic value in intensive care units (ICUs) as mSSEPs, demonstrating a specificity of $60 \%$ and a sensitivity of $47 \%$ at predicting poor outcomes after hypoxic-ischemic events. ${ }^{5}$ This may be due to the fact that the brainstem is less frequently affected than the cortex after cardiac arrest. ${ }^{6,7}$

\section{CASE REPORT}

A 34-year-old male was brought to the emergency department with a gunshot wound to the chest. Despite receiving 3 units of whole blood and multiple liters of intravenous fluids during his transport, the patient became hemodynamically unstable. Laboratory tests upon admission revealed as follows: $\mathrm{Hb} 7.3 \mathrm{~g} / \mathrm{dL}, \mathrm{Hct}$ 23\%, Na $149 \mathrm{mEq} / \mathrm{L}$, K $3.1 \mathrm{mEq} / \mathrm{L}$, Cl $114 \mathrm{mEq} / \mathrm{L}$, blood urea nitrogen $34 \mathrm{mg} / \mathrm{dL}$, Cr $1.2 \mathrm{mg} / \mathrm{dL}$; arterial gases (Fi 100\%): pH 6.57, $\mathrm{PaCO}_{2} 28, \mathrm{PaO}_{2} 216, \mathrm{HCO}_{3} 5$; toxicology screening: negative. Thus, from hypovolemic shock he subsequently experienced a cardiac arrest for 10 minutes before receiving a thoracotomy with direct heart compressions.

After return of spontaneous circulation, the patient was taken to the operating room for repair of his injuries and closure of his thoracotomy. Upon arrival to the ICU he was still intubated and on full mechanical ventilation, with no active bleeding. He was maintained under the effects of general anesthetics and neuromuscular junction blockers.

A non-contrasted head computed tomography showed mild signs of bilateral supra-tentorial brain edema. Differential diagnoses included 1) early manifestation of hypoxic-ischemic encephalopathy during the cardiac arrest, advanced reanimation, or surgical times; 2) positive fluid balance during the initial hospital stay; or 3) closed head trauma. Treatment was initiated with elevation of the head and midline neck positioning. In addition, he received a mannitol bolus $(1 \mathrm{~g} / \mathrm{kg})$. Due to concerns for potential coagulopathy from resuscitation, the patient was not treated with therapeutic hypothermia, and was maintained at $37^{\circ} \mathrm{C}$. He was sedated with propofol and monitored with continuous electroencephalography for 24 hours.

Over the next 2 days, the patient was weaned off vasopressor support and maintained a positive fluid balance. He continued to receive general supportive measures in the ICU without complications. Forty-eight hours after all sedatives and neuromuscular agents had been discontinued, he remained comatose. He was unresponsive to verbal or painful stimuli but demonstrated brainstem reflexes of direct and consensual photo-motors, corneal and gag. He had increased deep tendon reflexes $(+++/++)$ in a generalized fashion and bilateral Babinski's sign. On his 5th hospital day, mSSEPs and BAEPs for outcome prognostication were performed and are shown as Fig. 1.

After mSSEPs and BAEPs, arrangements were made with the patient's family to discuss prognosis and goals of care. Due to logistical issues the meeting was delayed for 2 days. On the 7th hospital day, the patient began to follow commands reliably, first with his lower and then upper extremities. Repeat mSSEPs were obtained that day and are shown as Fig. 2A, B. Because the N20 response of the left hemisphere was still not normal, a final noncontrasted and a perfusion single photon emission computed tomographies were ordered and are shown as Fig. 2C, D.

The patient survived and was released on the 10th hospital day, with persistent weakness of his upper extremities. A diagnosis of post-shock man in the barrel syndrome with anterior watershed infarctions, and a left area of posterior brain hypo-perfusion was made. His cerebral performance category was 3 and the modified Rankin score 4 on discharge.

\section{DISCUSSION}

To provide a clearer picture of the role the neurophysiological tests played in the patient's approach we consider a summary of the techniques used to collect them will help. The median nerves were independently stimulated at the ventral aspect of each wrist. Disposable surface electrodes were used with the cathode proximal to avoid anodal blockage. Constant, supra-threshold current square waves at $3.43 \mathrm{~Hz}$ frequency and $0.2 \mathrm{~ms}$ duration were delivered, 

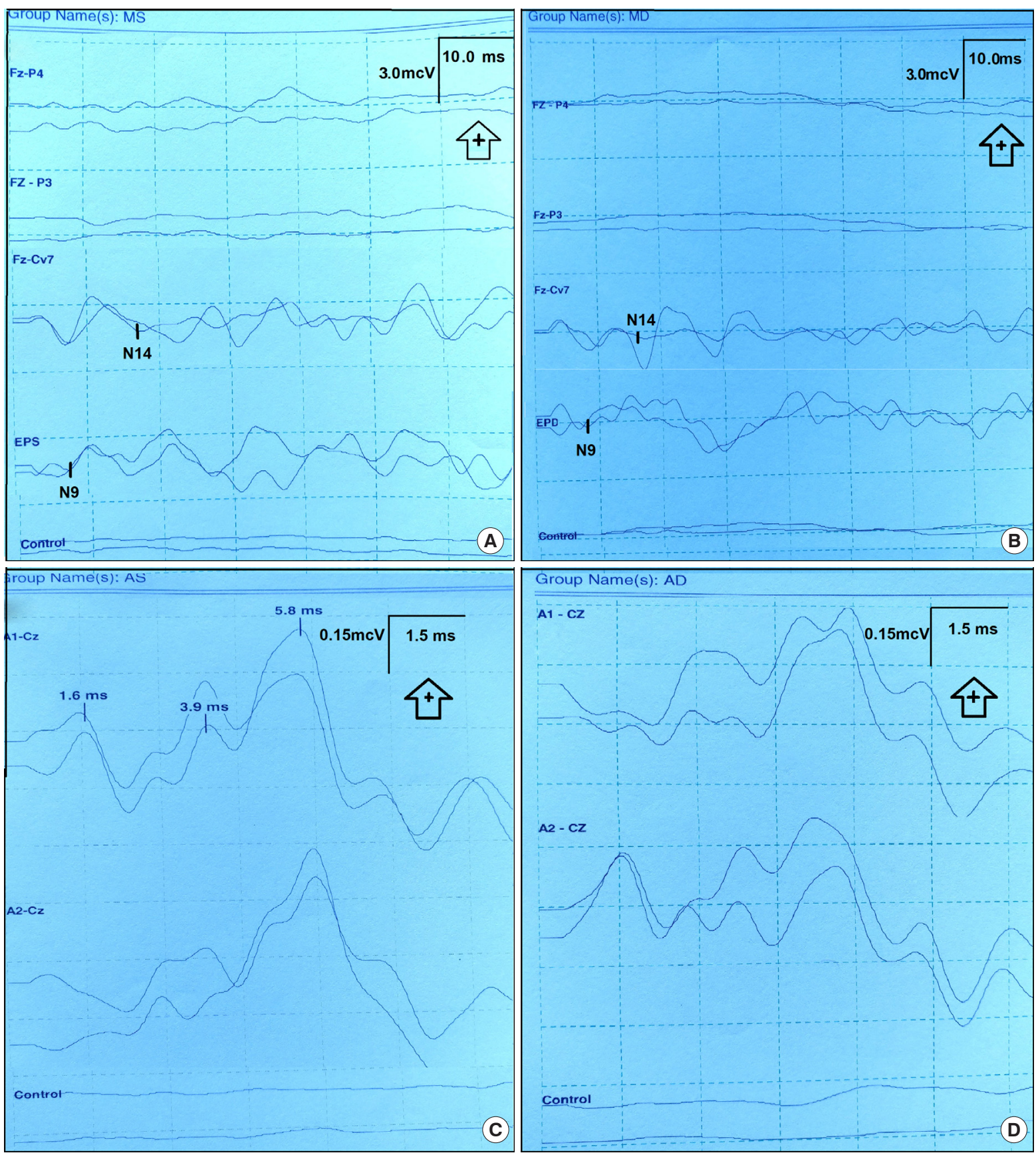

Fig. 1. Sensory evoked potentials' evaluation (5th hospital day). (A) Left median (MS) group: MS nerve somatosensory evoked potential. (B) Right median (MD) group: MD nerve somatosensory evoked potential. (C) Left ear (AS) group: left auditory evoked potential. (D) Right ear (AD) group: right auditory evoked potential. Fz, P4, P3, A1, A2, Cz: corresponding sites from the modified 10-20 international system of scalp electrode positioning. Cv7: spinous process of the 7th cervical vertebra; in this case the mastoid apophysis was used instead. The arrows point signals' polarity (inside). EPS, left Erb's point; $E P D$, right Erb's point.

once motor thresholds were obtained, confirmed by active hands flexion in a patient free of neuromuscular blockers. Two 512 trial epochs were collected through standard, disposable, scalp subdermal, mono-polar, steel needle electrodes. The N20/P30 cortical response was recorded from the ipsilateral and contralateral Parietal areas referenced to the midline Frontal site (P4/Fz and P3/Fz) from the modified 10-20 international system. The mastoid apophysis also referenced to the same site $(\mathrm{M} / \mathrm{Fz})$ was used instead of a spinous process of a cervical vertebra, to record the subcortical cervical (N13/P14) component of the upper extremity mSSEP, to avoid moving the patient from supine. Both Erb's points referenced to each other (left Erb's point/right Erb's point) 

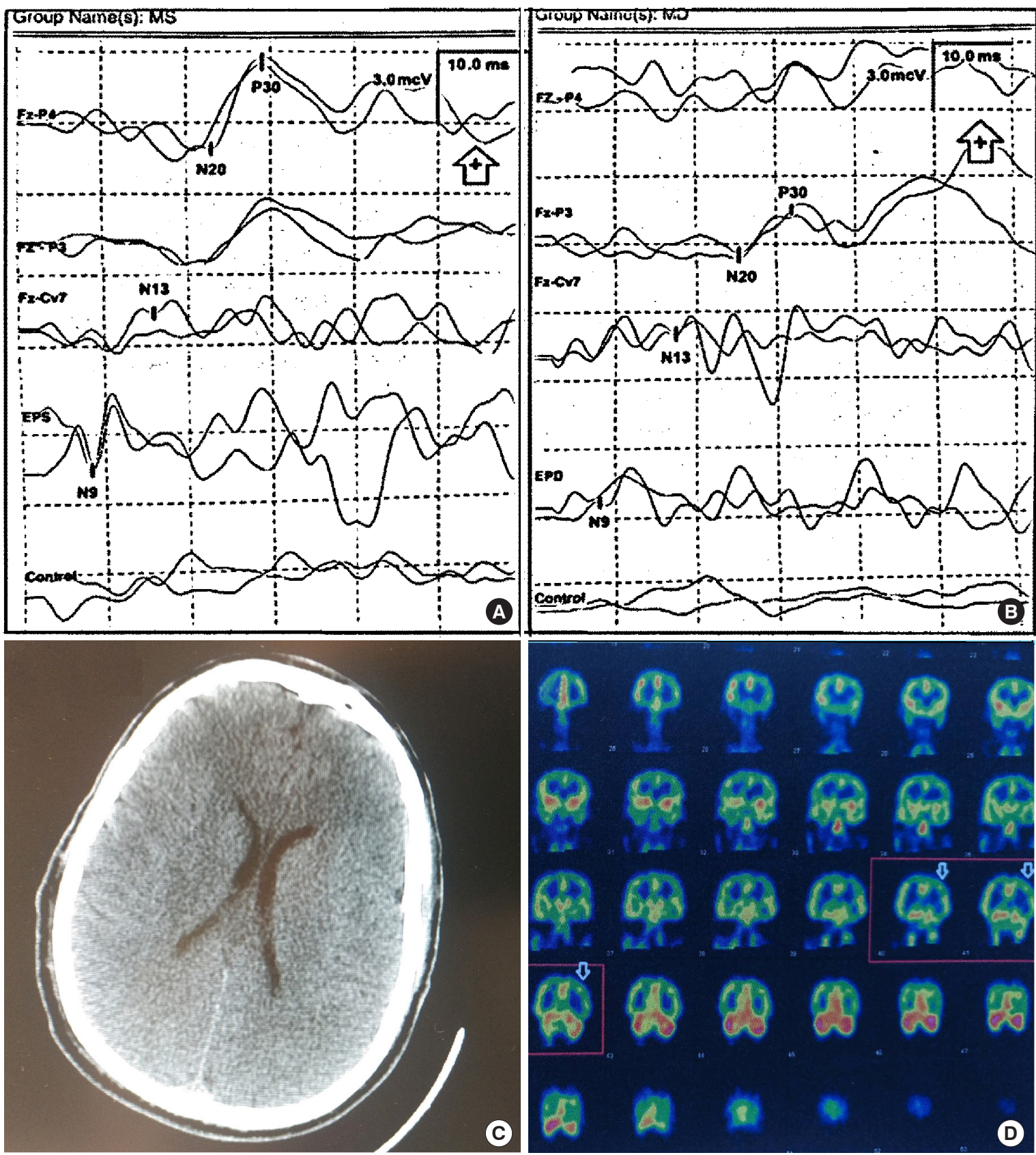

Fig. 2. Second median nerve somatosensory evoked potentials' evaluation (7th hospital day). (A) Left median (MS) group: MS nerve somatosensory evoked potential. (B) Right median (MD) group: MD nerve somatosensory evoked potential. UE: Fz, P4, P3: corresponding sites from the modified 10-20 international system of scalp electrode positioning. Cv7: spinous process of the 7th cervical vertebra; in this case the mastoid a pophysis was used instead. The arrows point signals' polarity (inside). (C) Non contrasted head computed tomography: axial cut showing bilateral, anterior watershed area's infarctions. (D) Head Tc-99-HMPAO computed tomography perfusion: coronal cuts note the left parietal brain area with hypoperfusion (arrows) inside the red box. EPS, left Erb's point; EPD, right Erb's point.

were used to collect the (N9/P10) peripheral responses. The bandpass filtering was 5 to $250 \mathrm{~Hz}$ with a $20 \mathrm{~K}$ gain for channels recording cortical responses, while 30 to $1,000 \mathrm{~Hz}$ with a gain of 50 $\mathrm{K}$ for sub-corticals. Five (5) $\mathrm{K} \Omega$ was the maximum electrode impedance accepted. This montage resulted in a slight variation from that recommended by the International Federation of Clinical Neurophysiology for the clinical practice of upper extremity mSSEPs. ${ }^{8}$

BAEPs were elicited by using sound clicks of $50 \mathrm{~ms}$ of monoaural stimulation carried out through ear inserts at $95 \mathrm{~dB}-\mathrm{HL}$ in- 
tensity and $17.6 \mathrm{~Hz}$ frequency. Alternating polarity (rarefaction/ condensation) was used to reduce stimulus artifact and to cancel cochlear microphonics. The contralateral ear was stimulated at $65 \mathrm{~dB}-\mathrm{HL}$ with white noise for masking purposes. Their recording was performed through standard, disposable, steel, mono-polar needle electrodes sub-dermally located at both ear lobes referenced to the vertex $(A 1 / C z$ and $A 2 / C z)$, from the modified $10-20$ international system. Bandpass filtering used was 100 to 1,000 $\mathrm{Hz}$ with a $100 \mathrm{~K}$ gain. A thousand and twenty-four trials were averaged per epoch of two collected, and electrode impedances of $5 \mathrm{~K} \Omega$, the maximum accepted. This montage also resulted in a very similar one to that recommended by the International Federation of Clinical Neurophysiology for the clinical practice of BAEPs. ${ }^{9}$

Anesthetic or sedative agents and low room temperature affect the quality of evoked potential recordings in ICUs. Although our patient was not treated with therapeutic hypothermia and was kept euthermic during his ICU stay, he received a propofol infusion. Propofol does not typically affect SSEPs' amplitude when used at doses of less than $2.5 \mathrm{mg} / \mathrm{kg}$. Likewise, it does not affect BAEPs' amplitudes when used at concentrations between 10 and $50 \mu \mathrm{g} / \mathrm{kg} \cdot \mathrm{min}^{4}{ }^{4}$ During critical illness states, propofol's metabolism can extend to 40 minutes. ${ }^{10}$ Thus, with normal initial BAEPs' recordings (Fig. $1 C, D$ ), we expected no effect of propofol in our patient's mSSEPs. Other circumstances in which initial bilateral absence of $\mathrm{N} 20$ responses from mSSEPs have been reported with survival or good neurological outcome are reversible hypoglycemic coma, viral encephalitis, carbamazepine intoxication, head trauma, and hemispheric infarction. ${ }^{11-13}$ These were not present in our patient. The N20 waveform at the Left Parietal areas did not have a completely normal configuration in the second evaluation of this patient (Fig. 2B) suggesting a concurrent degree of cerebral hypoperfusion, also present at posterior watershed areas, consistent with a man in the barrel syndrome, in which patients are affected mostly in anterior watershed areas (Fig. 2C), as demonstrated by the predominant motor compromise of the upper extremities in this patient. ${ }^{14}$ For all these reason, when discussing validity of neurophysiological studies, their reliability should always be mentioned.

The only existing studies have shown moderate inter-rater agreement, with average kappa levels of 0.30 to $0.72,{ }^{12,15}$ attributed mainly to noise in the signals. Noise was present in this case, predominantly in the neck and upper thoracic channels where electromyographic artifact is more frequent during examination in the supine patient not under neuromuscular blockade. Intra-rater reliability has better agreement with kappa levels of $0.84 .^{12}$ Standardizing techniques, obtaining high signal-to-noise ratios, using appropriate filtering, and averaging, are still useful solutions to obtain valid results. ${ }^{15}$ The limitations of only one evaluation with this electrophysiological test against several longitudinal observations, as well as, information obtained by different modalities of neurological evaluation, such as perfusion studies must be considered when establishing the prognosis for an individual patient. Thus, our case adds to the literature supporting multi-modal strategies for determining outcomes in post cardiac arrest patients.

\section{CONFLICT OF INTEREST}

No potential conflict of interest relevant to this article was reported.

\section{ACKNOWLEDGMENTS}

The authors wish to thank the support of the Center for Clinical Neurophysiology technical personnel from UPMC for their help with the collection of the evoked potentials on this patient.

Dr. Jon Rittenberger, MD, MS, Associate Professor of the University of Pittsburgh's Department of Emergency Medicine has received funding from the American Heart Association, Mallinkrodt, LLC, and BrainCools, LLC.

\section{REFERENCES}

1. Lee $Y C$, Phan TG, Jolley DJ, Castley HC, Ingram DA, Reutens DC. Accuracy of clinical signs, SEP, and EEG in predicting outcome of hypoxic coma: a meta-analysis. Neurology 2010;74: 572-80.

2. Zandbergen EG, de Haan RJ, Stoutenbeek CP, Koelman JH, Hijdra A. Systematic review of early prediction of poor outcome in anoxic-ischaemic coma. Lancet 1998;352:1808-12.

3. Bender A, Howell K, Frey M, Berlis A, Naumann M, Buheitel G. Bilateral loss of cortical SSEP responses is compatible with good outcome after cardiac arrest. J Neurol 2012;259:2481-3.

4. Banoub M, Tetzlaff JE, Schubert A. Pharmacologic and physiologic influences affecting sensory evoked potentials: implications for perioperative monitoring. Anesthesiology 2003;99: 716-37.

5. Guerit JM, de Tourtchaninoff M, Soveges L, Mahieu P. The prognostic value of three-modality evoked potentials (TMEPs) in anoxic and traumatic comas. Neurophysiol Clin 1993;23:20926.

6. Brierley JB, Graham DI, Adams JH, Simpsom JA. Neocortical death after cardiac arrest: a clinical, neurophysiological, and neuropathological report of two cases. Lancet 1971;298:560-5. 
7. Revesz T, Geddes JF. Symmetrical columnar necrosis of the basal ganglia and brain stem in an adult following cardiac arrest. Clin Neuropathol 1988;7:294-8.

8. Cruccu G, Aminoff MJ, Curio G, et al. Recommendations for the clinical use of somatosensory-evoked potentials. Clin Neurophysiol 2008;119:1705-19.

9. Pratt H, Aminoff M, Nuwer MR, Starr A. Short-latency auditory evoked potentials. The International Federation of Clinical Neurophysiology. Electroencephalogr Clin Neurophysiol Suppl 1999;52:69-77.

10. Marik PE. Propofol: therapeutic indications and side-effects. Curr Pharm Des 2004;10:3639-49.

11. Appoloni O, Mavroudakis N, Sadis C, Vincent JL. Reversible hypoglycemic coma despite bilateral absence of the median nerve
N20 evoked potential. Neurology 2003;60:1723-4.

12. Hakimi K, Kinney G, Kraft G, Micklesen P, Robinson L. Reliability in interpretation of median somatosensory evoked potentials in the setting of coma: factors and implications. Neurocrit Care 2009;11:353-61.

13. Schwarz S, Schwab S, Aschoff A, Hacke W. Favorable recovery from bilateral loss of somatosensory evoked potentials. Crit Care Med 1999;27:182-7.

14. Shaw PJ, Tharakaram S, Mandal SK. Brachial diplegia as a sequel to cardio-respiratory arrest: man-in-the-barrel syndrome. Postgrad Med J 1990;66:788.

15. Zandbergen EG, Hijdra A, de Haan RJ, et al. Interobserver variation in the interpretation of SSEPs in anoxic-ischaemic coma. Clin Neurophysiol 2006;117:1529-35. 